\title{
Endoscopic and Percutaneous Biliary Interventions after Liver Transplantation: Nationwide Data in Korea
}

\author{
Seung Bae Yoon ${ }^{1}$, Jungmee Kim², Chang Nyol Paik³ ${ }^{3}$ Dong Kee Jang ${ }^{4}$, Jun Kyu Lee ${ }^{4}$, Won Jae Yoon ${ }^{5}$, Jung-Wook \\ $\mathrm{Kim}^{6}$, Tae Hee Lee ${ }^{7}$, and Jae-Young Jang ${ }^{6}$ \\ ${ }^{1}$ Department of Internal Medicine, Eunpyeong St. Mary's Hospital, College of Medicine, The Catholic University of Korea, ${ }^{2}$ Department \\ of Preventive Medicine, Seoul National University College of Medicine, Seoul, ${ }^{3}$ Department of Internal Medicine, St. Vincent's \\ Hospital, College of Medicine, The Catholic University of Korea, Suwon, ${ }^{4}$ Department of Internal Medicine, Dongguk University Ilsan \\ Hospital, Dongguk University College of Medicine, Goyang, ${ }^{5}$ Department of Internal Medicine, Ewha Womans University College of \\ Medicine, ${ }^{6}$ Department of Internal Medicine, Kyung Hee University Hospital, Kyung Hee University College of Medicine, and ${ }^{7}$ Institute \\ for Digestive Research, Soonchunhyang University Seoul Hospital, Soonchunhyang University College of Medicine, Seoul, Korea
}

See editorial on page 145.

\section{Article Info}

Received December 28, 2020

Revised April 13, 2021

Accepted April 21, 2021

Published online July 12, 2021

\section{Corresponding Author}

Chang Nyol Paik

ORCID https://orcid.org/0000-0002-3470-6904

E-mail cmcu@catholic.ac.kr

\section{Dong Kee Jang}

ORCID https://orcid.org/0000-0001-6642-6635

E-mail mapmotive@hanmail.net

Seung Bae Yoon and Jungmee Kim contributed equally to this work as first authors.
Background/Aims: Biliary complications including biliary strictures and bile leaks are the most common complications that occur after liver transplantation (LT). Endoscopic treatment with endoscopic retrograde cholangiography (ERC) is considered the first-line treatment, and percutaneous transhepatic cholangiography (PTC) can serve as an alternative or rescue therapy. However, nationwide clinical data on the frequency of ERC and PTC following LT have not yet been investigated.

Methods: Using the nationwide claims database, we investigated patients who underwent LT between 2012 and 2014 in Korea and followed them until 2015. We analyzed the prevalence and characteristics of patients and biliary procedures, including ERC and PTC implemented after LT.

Results: A total of 3,481 patients underwent LT during the 3-year study period. Among them, $3.0 \%$ of patients underwent biliary intervention postoperatively during the same hospitalization period, and $21.4 \%$ of patients received biliary intervention later on after initially being discharged from the hospital following LT. A total of $16.9 \%$ and $12.1 \%$ of patients underwent ERC and PTC after $L T$, respectively. The median period from $L T$ to the first biliary intervention was 7.8 months (interquartile range, 3.5 to 14.6 months), and these patients underwent an average of $3.2 \pm 2.8$ biliary procedures during the follow-up period. Patients undergoing living donor LT were more than twice as likely to undergo biliary procedures as those undergoing deceased donor LT ( $25.5 \%$ vs $12.1 \%$ ).

Conclusions: Approximately one-fourth of patients in Korea who underwent LT subsequently underwent ERC or PTC. Compared with deceased donor LT patients, those undergoing living donor LT underwent more biliary interventions and were more difficult to treat. (Gut Liver 2022;16:300-307)

Key Words: Liver transplantation; Biliary duct disease; Cholangiopancreatography, endoscopic retrograde; Big data

\section{INTRODUCTION}

Liver transplantation (LT) is the main treatment for patients with end-stage liver disease, including liver failure and hepatocellular carcinoma. ${ }^{1}$ Despite improvements in surgical technique and experience, biliary complications still occur in about $20 \%$ to $40 \%$ of transplanted patients, and account for a major cause of morbidity and mortality after $\mathrm{LT}^{2-6}$ Common biliary complications are anastomotic or non-anastomotic biliary strictures, bile leaks, bile duct stones, bile casts, bilomas, and hemobilia. Although living donor LT (LDLT) offers several advantages over deceased donor LT (DDLT) and has evolved significantly worldwide, biliary complications after LDLT are known to be more 
frequent and more challenging. ${ }^{4,7-9}$

Early identification and proper management of biliary complications can help improve graft survival. ${ }^{6}$ Due to improvements in endoscopic tools and techniques, endoscopic retrograde cholangiography (ERC) is currently accepted as the first-line treatment for biliary complications after LT. ${ }^{8,10-12}$ If treatment through ERC is insufficient or impossible, percutaneous transhepatic cholangiography (PTC) can be the next option. However, there are no clear guidelines for treatment methods or sequences when the biliary complications occur.

Various regions and institutions have reported the prevalence and therapeutic outcomes of biliary complications following LT. ${ }^{3,12-14}$ However, nationwide clinical data regarding post-LT biliary interventions have not been assessed. In South Korea, the government operates a mandatory national health insurance system with a central database called the Health Insurance Review and Assessment (HIRA) service. The HIRA service in Korea covers claims for LT surgery as well as the endoscopic or percutaneous biliary interventions that follow. To evaluate post-LT biliary management, we conducted a nationwide cohort study using the HIRA database.

The primary endpoint of this study was to examine the prevalence of biliary interventions after LT in Korea. The secondary outcomes were the treatment modalities (ERC or PTC), timing and frequency of interventions, and biliary intervention-related factors in patients undergoing LT. In addition, the patients were largely divided into DDLT and LDLT groups to analyze the effects of LT method on biliary intervention.

\section{MATERIALS AND METHODS}

\section{Data sources}

The HIRA service operates under the Korean national insurance system and contains all insurance claims for the entire Korean population. ${ }^{15}$ For this reason, many epidemiologic analyses have been published from this database. The HIRA service provides data regarding each de-identified characteristic (e.g., sex and age), health-care service information (e.g., diagnosis, procedures, type of health-care service provider), and claim dates. LT surgery and biliary interventions including ERC and PTC that we assessed in this study were covered by the mandatory Korean insurance system. The Institutional Review Board of Eunpyeong St. Mary's Hospital approved this study (IRBnumber: PC20RCSI0192) and informed consents were waived.

\section{Subjects}

First, we identified all patients who received an LT between January 1, 2012 and December 31, 2014 by searching HIRA procedure codes for LT. Codes Q8040 to Q8044 correspond to DDLT, and codes Q8045 to Q8050 correspond to LDLT. In addition, through the procedure code numbers, we were able to identify the type of graft (e.g., Q8047 as LDLT with right liver).

For all patients who received an LT during the study period, follow-up was conducted for at least 1 year until December 31, 2015. During the follow-up period, all endoscopic or percutaneous biliary procedures were investigated using specific procedure codes in the HIRA data set (E7621 or Q7761-Q7767 for endoscopic biliary procedures and M6670, M6681, or M6682 for percutaneous biliary procedures). Endoscopic or percutaneous biliary procedures performed during the same hospitalization as the original LT surgery were excluded in our study. For convenience, endoscopic biliary procedures are named ERC and percutaneous biliary procedures are named PTC in this paper.

The institutional case volume was defined as the average annual number of total LTs. Institutions were categorized as low-volume ( $<10$ cases/year), medium-volume (10-50 cases/year), or high-volume ( $>50$ cases/year) centers based on the previous reference. ${ }^{16}$

\section{Statistical analysis}

Continuous data are presented as mean \pm standard deviation or median (interquartile range), and categorical data are presented as quantities and proportions. Comparisons of characteristics and variables between groups were performed by using the two-sample independent t-test or the Mann-Whitney $U$ test for numerical variables, and the Pearson chi-squared test for nominal variables. A logistic regression, where the presence of biliary interventions was a response variable and LT methods (DDLT vs LDLT) and institutional case volume (low-volume vs medium-volume vs high-volume) were explanatory variables, was calculated. Statistical analysis was performed using SAS version 9.3 (SAS Institute, Cary, NC, USA) and statistical significance was set at $\mathrm{p}<0.05$.

\section{RESULTS}

\section{LT in Korea}

According to data from HIRA, a total of 3,481 patients underwent LT surgeries in Korea between 2012 and 2014 (Fig. 1). Among them, 11 patients received more than two LT surgeries during the study period. This analysis includ- 
ed only the first LT and subsequent procedures.

Baseline characteristics of patients who underwent LT are shown in Table 1 . The mean age was $48.5 \pm 13.1$ years and $70.5 \%(2,453)$ were male. The median follow-up period in this study population was 30.3 months (interquartile range, 21.0 to 39.5 months). Patients of $29.9 \%(1,041 / 3,481)$ received DDLT, and the remaining $70.1 \%(2,440 / 3,481)$ received LDLT. The majority of DDLT patients received a whole liver (963/1,041, 92.5\%), while the majority of LDLT patients $(2,317 / 2,440,95.0 \%)$ received a right liver lobe.

\section{Biliary interventions during the same hospitalization as LT}

A total of 103 patients (3.0\%) received any biliary intervention including ERC and PTC during the same hospital-

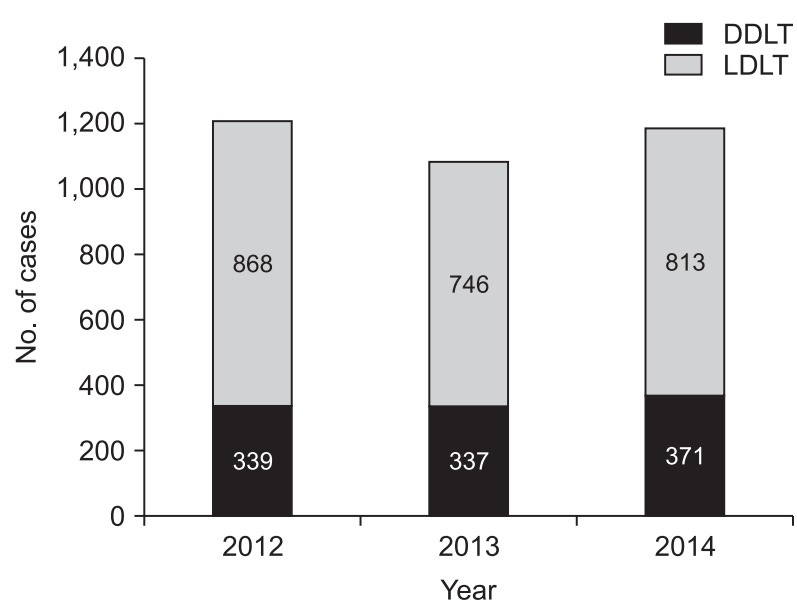

Fig. 1. The number of liver transplantations in Korea between 2012 and 2014.

DDLT, deceased donor liver transplantation; LDLT, living donor liver transplantation. ization as LT surgery (Table 1). Seventy-one (2.0\%) and 39 (1.1\%) patients received ERC and PTC, respectively. The proportions of patients undergoing biliary interventions during the same hospitalization as LT were comparable between DDLT and LDLT (3.0 vs 3.0\%, p=0.9).

\section{Biliary interventions after LT}

A total of 746 patients (21.4\%) received any biliary intervention including ERC and PTC after LT (Table 1). Five hundred and eighty-eight (16.9\%) and 422 (12.1\%) patients received ERC and PTC, respectively. The proportion of patients undergoing biliary interventions after LT was significantly higher in LDLT patients $(620 / 2,440,25.4 \%)$ than in DDLT patients $(126 / 1,041,12.1 \%, \mathrm{p}<0.001)$. The proportion of patients who received ERC after LT was about two times higher in LDLT compared to DDLT (19.9\% vs $9.9 \%$, p<0.001). LDLT patients received PTC after LT more than three times as often as DDLT patients $(15.4 \%$ vs $4.5 \%, \mathrm{p}<0.001)$.

Characteristics of biliary interventions after LT are shown in Table 2. The median period from LT to the first biliary intervention was 7.8 months (interquartile range, 3.5 to 14.6 months), and did not differ significantly between LDLT and DDLT. During the follow-up period, LDLT patients $(3.4 \pm 2.9)$ received more biliary interventions than DDLT patients $(2.6 \pm 2.5, \mathrm{p}=0.002)$. Of the 746 patients who received biliary interventions after LT, 324 (43.4\%) and 158 (21.2\%) patients were treated with ERC and PTC alone, respectively; the remaining 264 patients (35.4\%) were treated with a combination of both methods. The percentage of patients who were treated with ERC alone was significantly lower in LDLT (39.5\%) compared to DDLT patients $(62.7 \%$, $\mathrm{p}<0.001)$. On the other hand, LDLT patients were more likely to be treated with combined modalities (38.7\%) than

Table 1. Characteristics of Patients Who Underwent Liver Transplantation between 2012 and 2014 and Were Followed until 2015

\begin{tabular}{|c|c|c|c|c|}
\hline Parameter & Total $(n=3,481)$ & $\operatorname{DDLT}(n=1,041)$ & $\operatorname{LDLT}(n=2,440)$ & p-value \\
\hline Age, yr & $48.5 \pm 13.1$ & $47.7 \pm 14.6$ & $48.6 \pm 12.4$ & $<0.001$ \\
\hline Male sex & $2,453(70.5)$ & $683(65.6)$ & $1,770(72.5)$ & $<0.001$ \\
\hline Follow-up periods, day & $30.0(21.0-39.5)$ & $29.7(20.3-38.8)$ & $30.7(21.2-39.6)$ & 0.04 \\
\hline Graft & & & & $<0.001$ \\
\hline Whole liver & $963(27.7)$ & 963 (92.5) & - & \\
\hline Right liver & $2,350(67.5)$ & 33 (3.2) & $2,317(95.0)$ & \\
\hline Left liver & $168(4.8)$ & $45(4.3)$ & $123(5.0)$ & \\
\hline Any biliary interventions during the same hospitalization as LT & $103(3.0)$ & $31(3.0)$ & $72(3.0)$ & 0.9 \\
\hline ERC during same hospitalization as LT & $71(2.0)$ & $23(2.2)$ & $48(2.0)$ & 0.6 \\
\hline PTC during same hospitalization as LT & $39(1.1)$ & $9(0.9)$ & $30(1.2)$ & 0.3 \\
\hline Any biliary interventions after $L T$ & $746(21.4)$ & $126(12.1)$ & $620(25.4)$ & $<0.001$ \\
\hline ERC after LT & 588 (16.9) & 103 (9.9) & $485(19.9)$ & $<0.001$ \\
\hline PTC after LT & $422(12.1)$ & $47(4.5)$ & $375(15.4)$ & $<0.001$ \\
\hline
\end{tabular}

Data are presented as mean \pm SD, number (\%), or median (IQR).

LT, liver transplantation; DDLT, deceased donor LT; LDLT, living donor LT; ERC, endoscopic retrograde cholangiography; PTC, percutaneous transhepatic cholangiography; IQR, interquartile range. 
Yoon SB, et al: Biliary Interventions after Liver Transplantation

Table 2. Characteristics of Biliary Interventions after Liver Transplantation between 2012 and 2015

\begin{tabular}{lcccc}
\multicolumn{1}{c}{ Parameter } & Total $(\mathrm{n}=746)$ & DDLT $(\mathrm{n}=126)$ & LDLT (n=620) & $\mathrm{p}$-value \\
\hline Time to first biliary interventions after LT, mo & $7.8(3.5-14.6)$ & $8.8(3.4-15.0)$ & $7.7(3.5-14.6)$ & 0.3 \\
No. of total biliary interventions & $3.2 \pm 2.8$ & $2.6 \pm 2.5$ & $3.4 \pm 2.9$ & 0.002 \\
No. of ERCs & $2.1 \pm 2.3$ & $1.8 \pm 2.2$ & $2.1 \pm 2.3$ & 0.2 \\
No. of PTCs & $1.2 \pm 1.7$ & $0.7 \pm 1.7$ & $1.2 \pm 1.7$ & 0.003 \\
Only ERC after LT & $324(43.4)$ & $79(62.7)$ & $245(39.5)$ & $<0.001$ \\
Only PTC after LT & $158(21.2)$ & $23(18.3)$ & $135(21.8)$ & 0.4 \\
Both ERC and PTC after LT & $264(35.4)$ & $24(19.0)$ & $240(38.7)$ & $<0.001$ \\
ERC first, then PTC & $141(18.9)$ & $7(5.6)$ & $134(21.6)$ & $<0.001$ \\
PTC first, then ERC & $27(3.6)$ & $4(3.2)$ & $23(3.7)$ & 0.8 \\
Combined modalities simultaneously* & $96(12.9)$ & $13(10.3)$ & $83(13.4)$ & 0.4 \\
\hline
\end{tabular}

Data are presented as median (IQR), mean \pm SD, or number (\%).

LT, liver transplantation; DDLT, deceased donor LT; LDLT, living donor LT; ERC, endoscopic retrograde cholangiography; PTC, percutaneous transhepatic cholangiography; IQR, interquartile range.

${ }^{*}$ Combined modalities refer to both ERC and PTC being performed for initial biliary management during the same hospitalization.

Table 3. Comparison of Characteristics between Patients with and without Biliary Interventions after Liver Transplantation between 2012 and 2015

\begin{tabular}{|c|c|c|c|}
\hline Parameter & Biliary interventions ( $n=746$ ) & No biliary interventions $(n=2,735)$ & $\mathrm{p}$-value \\
\hline Age, yr & $50.2 \pm 10.6$ & $48.2 \pm 13.7$ & $<0.001$ \\
\hline Male sex & $538(72.1)$ & $1,915(70.0)$ & 0.2 \\
\hline Graft & & & $<0.001$ \\
\hline DDLT & $124(16.6)$ & $918(33.6)$ & \\
\hline LDLT, right liver & 603 (80.8) & $1,713(66.5)$ & \\
\hline LDLT, left liver & $19(2.5)$ & 104 (3.5) & \\
\hline
\end{tabular}

Data are presented as mean \pm SD or number $(\%)$.

DDLT, deceased donor liver transplantation; LDLT, living donor liver transplantation.

DDLT patients $(19.0 \%, \mathrm{p}<0.001)$. Patients who received both ERC and PTC most frequently received ERC first, followed by PTC $(53.4 \%, 141 / 264)$. In addition, LDLT patients were more likely to receive ERC first, the PTC compared to DDLT patients ( $21.6 \%$ vs $5.6 \%$, $\mathrm{p}<0.001)$.

\section{Comparison of characteristics between patients with and without biliary interventions}

Patients who received biliary intervention after LT (mean age, $50.2 \pm 10.6$ years) were older than those without biliary interventions $(48.2 \pm 13.7$ years, $\mathrm{p}<0.001)$ (Table 3 ). There was no significant sex difference between the two groups. About four-fifths $(80.8 \%)$ of patients who underwent biliary interventions after LT received the graft from the right liver of the living donor, and this was significantly higher than those who did not undergo biliary interventions $(66.5 \%, \mathrm{p}<0.001)$.

\section{Effect of institutional case volume of LT on biliary intervention}

LT surgeries were performed at 53 centers during the study period (Table 4). Low-volume (36 centers), mediumvolume (seven centers), and high-volume (10 centers) centers performed 350 (10.1\%), 410 (11.8\%), and 2,721 (78.2\%) cases, respectively. DDLT was higher $(61.4 \%)$ in low-volume centers, while LDLT accounted for the majority $(77.4 \%)$ of LTs in high-volume centers $(p<0.001)$. The crude prevalence of patients who received biliary intervention after LT did not differ significantly according to institutional volume $(20.6 \%$ in low-volume, $19.3 \%$ in medium, and $21.8 \%$ in high-volume centers, respectively, $\mathrm{p}=0.461$ ).

Multivariable logistic regression analysis accounting for LT methods and institutional volumes showed that LDLT was related to a higher frequency of biliary interventions (adjusted odds ratio, 2.7; $\mathrm{p}<0.001$ ) (Table 5). In addition, patients receiving LT at high-volume centers had a relatively lower rate of biliary intervention than those at smallvolume centers (adjusted odds ratio, $0.7 ; \mathrm{p}=0.05$ ).

\section{DISCUSSION}

This study evaluated nationwide clinical data for biliary interventions including ERC and PTC conducted after LT in Korea. Overall, about one-fifth of patients receiving LT underwent biliary interventions during the follow-up period. Patients who received LDLT had a higher rate of biliary interventions, as well as a higher number of procedures and additional PTCs. Type of liver graft and institutional case volume of LT affected the prevalence of biliary inter- 
Table 4. Biliary Interventions According to Medical Institution Classified by the Volume of Liver Transplantation Cases between 2012 and 2014

\begin{tabular}{|c|c|c|c|c|c|}
\hline Parameter & Total & $\begin{array}{l}\text { Low-volume } \\
\text { (<10 cases/year) }\end{array}$ & $\begin{array}{l}\text { Medium-volume } \\
\text { (10-50 cases/year) }\end{array}$ & $\begin{array}{l}\text { High-volume } \\
\text { (>50 cases/year) }\end{array}$ & p-value \\
\hline No. of institutions & 53 & 36 (67.9) & 7 (13.2) & 10 (18.9) & \\
\hline Total number of LT cases & 3,481 & $350(10.1)$ & $410(11.8)$ & $2,721(78.2)$ & \\
\hline Annual number of LTs per institution & & $5.1 \pm 7.3$ & $35.5 \pm 42.3$ & $198.8 \pm 368.0$ & \\
\hline Types of LT & & & & & $<0.001$ \\
\hline DDLT & 1,041 & $215(61.4)$ & 210 (51.2) & $616(22.6)$ & \\
\hline LDLT & 2,440 & $135(38.6)$ & $200(48.8)$ & $2,105(77.4)$ & \\
\hline Biliary interventions after LT ( $\%$ of total LTs) & $745(21.4)$ & $72(20.6)$ & 79 (19.3) & $594(21.8)$ & 0.5 \\
\hline ERC after LT (\% of total LTs) & $588(16.9)$ & $58(16.6)$ & $62(15.1)$ & 468 (17.2) & 0.6 \\
\hline PTC after LT ( $\%$ of total LTs) & $422(12.1)$ & $38(10.9)$ & $43(10.5)$ & $341(12.5)$ & 0.4 \\
\hline Both ERC and PTC after LT (\% of total LTs) & $265(7.6)$ & $24(6.9)$ & $26(6.3)$ & $215(7.9)$ & 0.5 \\
\hline
\end{tabular}

Data are presented as number (\%) or mean \pm SD.

LT, liver transplantation; DDLT, deceased donor LT; LDLT, living donor LT; ERC, endoscopic retrograde cholangiography; PTC, percutaneous transhepatic cholangiography.

Table 5. Odds Ratios for Undergoing Biliary Intervention after Liver Transplantation

\begin{tabular}{lcc}
\hline \multicolumn{1}{c}{ Variable } & aOR $(95 \% \mathrm{Cl})$ & $\mathrm{p}$-value \\
\hline DDLT & Reference & \\
LDLT & $2.7(2.2-3.4)$ & $<0.001$ \\
$\begin{array}{l}\text { LT-performed hospital } \\
\text { Low-volume }\end{array}$ & Reference & \\
Medium-volume & $0.8(0.6-1.2)$ & 0.3 \\
High-volume & $0.7(0.6-1.0)$ & 0.05 \\
\hline
\end{tabular}

aOR, adjusted odds ratio; $\mathrm{Cl}$, confidence interval; LT, liver transplantation; DDLT, deceased donor LT; LDLT, living donor LT.

ventions after LT.

LT is the ultimate treatment for end-stage liver disease and the first DDLT was successfully implemented in Korea in $1988 .{ }^{17}$ Meanwhile, LDLT was first performed by Lee et al. in $1994,{ }^{18}$ and has become more popular especially as technologies have developed due to the limited supply of deceased donor liver grafts. There is a great demand for LT in Korea because of the high prevalence of hepatitis B viral infection and hepatocellular carcinoma. Since 2002, due to the revision of the brain death law and the promotion of various social campaign programs, organ donation has increased dramatically in Korea. In 2010, there were 23.2 LTs per million population in Korea, which was higher than the 20 per million population in the United States. ${ }^{17}$ In our study, more than one thousand patients received LT each year between 2012 and 2014, and the ratio of LDLT and DDLT was around 7:3. This situation in South Korea was suitable for analyzing and comparing the characteristics of biliary interventions according to LT method.

The reported prevalence of post-LT biliary complications varies widely from $5 \%$ to $45 \% .{ }^{19-21}$ Various factors including liver graft, LT type, and institutional capacity may account for this disparity. In our study, the proportion of patients receiving biliary procedures during same hospitalization as LT and after LT was 3.0\% and $21.4 \%$, respectively, which is within the ranges of biliary complication prevalence reported by major hospitals in Korea. ${ }^{3,22-24}$ Because ERC or PTC procedures are generally performed in the event of post-LT biliary complications, the approximate prevalence of bile duct complications may be extrapolated through the post-LT biliary intervention rates in this nationwide claim data.

ERC has become the first-line modality for the management of biliary complications after LT, especially in cases with anastomotic stenosis. ${ }^{25,26}$ Likewise, in our study, the preferred treatment method was ERC therapy, then followed by combined therapy with ERC and PTC, followed by therapy with PTC alone. In addition, among patients receiving combination ERC and PTC therapy, the majority of cases underwent ERC first and PTC later. Due to advances in ERC's technology and devices, ERC has become the main treatment modality even for the biliary complications of LDLT patients, which are relatively challenging to perform. The procedures through ERC are mainly composed of balloon dilatation followed by placement of multiple plastic stents. Recent studies suggested that covered self-expandable metal stents may be useful in LT patients with refractory biliary strictures, as these improve stricture resolution rates and reduce the number of ERC sessions required for stricture resolution. ${ }^{27,28}$

Meanwhile, PTC is usually reserved for cases in which an endoscopic approach is unsuccessful or impossible (i.e., Roux-en-Y hepaticojejunostomy status). For these intractable cases, percutaneous transhepatic biliary drainage and subsequent balloon dilation and/or indwelling catheter placement are valuable modalities. In our study, PTC was mainly performed in LDLT patients, and most were performed in combination with ERC. In addition, successful PTC sometimes can lead to subsequent ERC treatment using the rendezvous technique or adjuvant percutaneous 
transhepatic cholangioscopy for the management of postLT biliary complications. ${ }^{29,30}$

Bile leaks occur in the range of $2 \%$ to $15 \%$ and, most bile leaks are seen from 1 day to 4 weeks after LT. A metaanalysis showed that the prevalence of bile leaks in DDLT and LDLT was similar at $7.8 \%$ and $9.5 \%,{ }^{5}$ which was consistent in the finding that patients undergoing biliary interventions during the same hospitalization as LT were comparable between DDLT and LDLT in our study. Meanwhile, anatomic biliary strictures are the most common post-LT biliary complications and typically occur within 1 year following $\mathrm{LT}^{20}$ Accordingly, our study reported that the median time interval from LT to the first biliary intervention was 7.8 months. Therefore, in LT patients who have cholestasis within 1 year, the priority is to differentiate biliary stricture from liver parenchymal causes including rejection, recurrence of primary disease, and drug-induced hepatitis. In our study, those who underwent biliary interventions received an average of 3.2 sessions during the follow-up period, suggesting that post-LT biliary complications are generally overcome through repetitive procedures.

LDLT is considered an effective and feasible treatment of end-stage liver disease given a scarcity of deceased donor liver grafts, however, it is technically more complex and challenging. Biliary complications also arise more frequently in LDLT compared to DDLT due to the small duct size and the presence of multiple biliary duct outlets. ${ }^{6}$ The characteristics of biliary interventions in LDLT patients compared to DDLT were well demonstrated in our study. LDLT patients had a higher rate and number of overall biliary interventions, as well as a higher rate of combined ERC and PTC. Therefore, especially in countries with a high LDLT ratio, such as Korea, more active and comprehensive biliary interventions should be considered and implemented. Especially, in recent years, duct-to-duct biliary reconstruction has been preferred over hepaticojejunostomy, because of its simplicity, rapid gastrointestinal recovery, and preservation of physiological bilioenteric continuity. ${ }^{8,31}$ This situation allows more active endoscopic access to the biliary system for the management of biliary complications following LDLT. Additionally, LDLT patients generally received ERC first and PTC later, suggesting that majority of hospitals in Korea were using ERC as a first-line treatment and PTC as a rescue or alternative treatment for biliary interventions in LDLT patients.

A recent study in Korea reported that institutional case volume affected clinical outcomes after LDLT. ${ }^{16}$ Institutions with higher case volumes ( $>50$ cases/year) had better outcomes compared to those with lower case volumes ( $\leq 50$ cases/year). Similarly, our study found that patients who received LT at institutions with high case volume were less likely to receive biliary interventions than those at low case volume institutions. Differences in multidisciplinary approaches, as well as surgical experience, may have made this difference.

Our study has some limitations. First, we could not evaluate primary diseases, biliary reconstruction methods, and the outcomes including clinical success or mortality after biliary interventions in this study. Second, it was impossible to analyze the specific type of the biliary complications when the biliary interventions were performed. Third, the interval of biliary interventions and the number of rescue surgical procedures were not fully analyzed. Finally, because this study was based on a right-censored data, follow-up periods vary from 1 to 4 years.

Notwithstanding, the major strength of our study is that our results represent nearly all LT patients in the Korean population. Since there have been no clear standards or guidelines for management of biliary complications after LT, each institution might have different treatment criteria and processes. Our study could minimize the selection and recruitment biases which are inevitable in a cohort study, using data representative of the entire Korean population. LT surgery and subsequent biliary interventions are mostly covered by insurance in Korea. The number of LT cases of our study was more than $93 \%(3,492 / 3,741)$ consistent with the number of LTs announced by the Korean Network for Organ Sharing system, which supervises all types of organ donation, procurement, and transplantation in Korea. ${ }^{17}$ Furthermore, since the research was conducted using a nationwide database, there was no missing or censored data even though patients changed their health institution during the study period. Therefore, our results show the real clinical situation in Korea as a whole.

In conclusion, approximately one-fourth of patients who underwent LT subsequently received ERC or PTC in Korea. Compared to DDLT patients, patients undergoing LDLT received a higher percentage and number of biliary interventions and needed combination modalities including both ERC and PTC more frequently. Institutional case volume of LT might affect the prevalence of biliary complications after LT. The results of the present study indicate the common attitude to consider ERC as the firstline treatment for biliary complications after LT. Repetitive procedures are required in many cases, and PTC can play a role in alternative or rescue therapies.

\section{CONFLICTS OF INTEREST}

W.J.Y. is an editorial board member of the journal but 
was not involved in the peer reviewer selection, evaluation, or decision process of this article. No other potential conflicts of interest relevant to this article were reported.

\section{ACKNOWLEDGEMENTS}

This study was supported by a grant from the Korean Gastrointestinal Endoscopy Research Foundation (T.H.L.).

\section{AUTHOR CONTRIBUTIONS}

Conception and design of the study: S.B.Y., C.N.P., J.W.K., J.K.L. Generation, collection, assembly, analysis and/or interpretation of data: S.B.Y., J.K. Drafting of the manuscript: S.B.Y., C.N.P., W.J.Y. Obtaining funding: T.H.L. Revision of the manuscript: D.K.J. Study supervision: T.H.L., J.Y.J.

\section{ORCID}

Seung Bae Yoon

https://orcid.org/0000-0002-6119-7236

Jungmee Kim https://orcid.org/0000-0002-1955-843X

Chang Nyol Paik https://orcid.org/0000-0002-3470-6904

Dong Kee Jang https://orcid.org/0000-0001-6642-6635

Jun Kyu Lee https://orcid.org/0000-0002-2694-3598

Won Jae Yoon https://orcid.org/0000-0003-2292-1348

Jung-Wook Kim https://orcid.org/0000-0002-5383-7934

Tae Hee Lee https://orcid.org/0000-0003-3049-8252

Jae-Young Jang https://orcid.org/0000-0002-7930-1468

\section{REFERENCES}

1. Halliday N, Westbrook RH. Liver transplantation: need, indications, patient selection and pre-transplant care. Br J Hosp Med (Lond) 2017;78:252-259.

2. Rerknimitr R, Sherman S, Fogel EL, et al. Biliary tract complications after orthotopic liver transplantation with choledochocholedochostomy anastomosis: endoscopic findings and results of therapy. Gastrointest Endosc 2002;55:224-231.

3. Chang JH, Lee IS, Choi JY, et al. Biliary stricture after adult right-lobe living-donor liver transplantation with duct-toduct anastomosis: long-term outcome and its related factors after endoscopic treatment. Gut Liver 2010;4:226-233.

4. Kyoden Y, Tamura S, Sugawara Y, et al. Incidence and management of biliary complications after adult-to-adult living donor liver transplantation. Clin Transplant 2010;24:535542.
5. Akamatsu N, Sugawara Y, Hashimoto D. Biliary reconstruction, its complications and management of biliary complications after adult liver transplantation: a systematic review of the incidence, risk factors and outcome. Transpl Int 2011;24:379-392.

6. Wan P, Yu X, Xia Q. Operative outcomes of adult living donor liver transplantation and deceased donor liver transplantation: a systematic review and meta-analysis. Liver Transpl 2014;20:425-436

7. Gómez CM, Dumonceau JM, Marcolongo M, et al. Endoscopic management of biliary complications after adult living-donor versus deceased-donor liver transplantation. Transplantation 2009;88:1280-1285.

8. Tsujino T, Isayama H, Kogure H, Sato T, Nakai Y, Koike K. Endoscopic management of biliary strictures after living donor liver transplantation. Clin J Gastroenterol 2017;10:297311.

9. Lee HW, Shah NH, Lee SK. An update on endoscopic management of post-liver transplant biliary complications. Clin Endosc 2017;50:451-463.

10. Larghi A, Tringali A, Rimbaş M, et al. Endoscopic management of benign biliary strictures after liver transplantation. Liver Transpl 2019;25:323-335.

11. Crismale JF, Ahmad J. Endoscopic management of biliary issues in the liver transplant patient. Gastrointest Endosc Clin N Am 2019;29:237-256.

12. Cantù P, Parzanese I, Balassone V, et al. Management of biliary anastomotic strictures after liver transplantation (BASALT study): a nationwide Italian survey. Liver Transpl 2017;23:257-261.

13. Humar A, Ganesh S, Jorgensen D, et al. Adult living donor versus deceased donor liver transplant (LDLT versus DDLT) at a single center: time to change our paradigm for liver transplant. Ann Surg 2019;270:444-451.

14. Cantù $\mathrm{P}$, Tarantino I, Baldan A, et al. Endo-therapies for biliary duct-to-duct anastomotic stricture after liver transplantation: outcomes of a nationwide survey. Liver Int 2019;39:1355-1362.

15. Kim JA, Yoon S, Kim LY, Kim DS. Towards actualizing the value potential of Korea Health Insurance Review and Assessment (HIRA) data as a resource for health research: strengths, limitations, applications, and strategies for optimal use of HIRA data. J Korean Med Sci 2017;32:718-728.

16. Yoo S, Jang EJ, Yi NJ, et al. Effect of institutional case volume on in-hospital mortality after living donor liver transplantation: analysis of 7073 cases between 2007 and 2016 in Korea. Transplantation 2019;103:952-958.

17. Lee SG, Moon DB, Hwang S, et al. Liver transplantation in Korea: past, present, and future. Transplant Proc 2015;47:705-708.

18. Lee SG, Lee YJ, Park KM, et al. Living related donor liver 
transplantation: the Seoul experience. Transplant Proc 1996;28:2383-2384.

19. Brown RS Jr, Russo MW, Lai M, et al. A survey of liver transplantation from living adult donors in the United States. N Engl J Med 2003;348:818-825.

20. Balderramo D, Navasa M, Cardenas A. Current management of biliary complications after liver transplantation: emphasis on endoscopic therapy. Gastroenterol Hepatol 2011;34:107115.

21. Kochhar G, Parungao JM, Hanouneh IA, Parsi MA. Biliary complications following liver transplantation. World J Gastroenterol 2013;19:2841-2846.

22. Hwang S, Lee SG, Sung KB, et al. Long-term incidence, risk factors, and management of biliary complications after adult living donor liver transplantation. Liver Transpl 2006;12:831838.

23. You MS, Paik WH, Choi YH, et al. Optimal biliary drainage for patients with biliary anastomotic strictures after right lobe living donor liver transplantation. Liver Transpl 2019;25:1209-1219.

24. Park JK, Yang JI, Lee JK, et al. Long-term outcome of endoscopic retrograde biliary drainage of biliary stricture following living donor liver transplantation. Gut Liver 2020;14:125134.

25. Tringali A, Barbaro F, Pizzicannella M, et al. Endoscopic management with multiple plastic stents of anastomotic biliary stricture following liver transplantation: long-term results. Endoscopy 2016;48:546-551.

26. Tarantino I, Amata M, Cicchese N, et al. Sequential multistenting protocol in biliary stenosis after liver transplantation: a prospective analysis. Endoscopy 2019;51:1130-1135.

27. Jang SI, Sung SY, Park H, Lee KH, Joo SM, Lee DK. Salvage therapy using self-expandable metal stents for recalcitrant anastomotic strictures after living-donor liver transplantation. Therap Adv Gastroenterol 2017;10:297-309.

28. Martins FP, De Paulo GA, Contini MLC, Ferrari AP. Metal versus plastic stents for anastomotic biliary strictures after liver transplantation: a randomized controlled trial. Gastrointest Endosc 2018;87:131.e1-131.e13.

29. Chang JH, Lee IS, Chun HJ, et al. Usefulness of the rendezvous technique for biliary stricture after adult right-lobe living-donor liver transplantation with duct-to-duct anastomosis. Gut Liver 2010;4:68-75.

30. Nam K, Lee SK, Song TJ, et al. Percutaneous transhepatic cholangioscopy for biliary complications after liver transplantation: a single center experience. J Hepatobiliary Pancreat Sci 2016;23:650-657.

31. Lee DW, Han J. Endoscopic management of anastomotic stricture after living-donor liver transplantation. Korean J Intern Med 2019;34:261-268. 